\section{Visión Electrónica Más que un estado sólido \\ https://doi.org/10.14483/issn.2248-4728}

UNIVERSIDAD DISTRITAL

FRANCISCO JOSÉ DE CALDAS

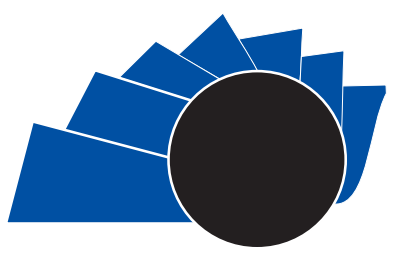

VISIÓN ELECTRONICA

Visión de caso

\title{
Recolector autónomo de bolas de tenis mediante visión artificial
}

\author{
Autonomous collector of tennis balls through artificial vision
}

John Alejandro Forero Casallas ${ }^{1}$, David Leonardo Gaitán Tabares ${ }^{2}$, Héctor Fabio Martínez Arcila ${ }^{3}$

\section{INFORMACIÓN DEL ARTICULO}

Historia del articulo Enviado: 16/01/2018

Recibido: 24/03/2018

Aceptado: 31/05/2018

Palabras clave:

LabVIEW

Lego

Robótica

Visión Artificial

Keywords:

LabVIEW

Lego

Robotic

Artificial Vision

\section{RESUMEN}

El artículo presenta el diseño, la programación y la construcción de un sistema autónomo para la recolección de pelotas de tenis utilizando un dispositivo de visión artificial y la plataforma robótica programable Lego ${ }^{\circledR}$ Mindstorms, cuya aplicación principal es levantar las bolas que quedan ubicadas en la periferia de la cancha durante los juegos entre dos personas (singles), o más competidores (doubles). El objetivo del sistema elaborado es evitar que los jugadores o asistentes del juego, estén desplazándose de un lugar a otro a recoger las pelotas que quedan esparcidas en la cancha, y en lugar de ir ellos enviar al robot desde cierta distancia. Para el desarrollo del proyecto se llevó a cabo la codificación del sistema en el lenguaje de programación suministrado por el software LabVIEW ${ }^{\circledR}$ de la compañía norteamericana National Instruments.

\section{ABSTRACT:}

The article presents the design, programming and construction of an autonomous system for the collection of tennis balls using an artificial vision device and the programmable robotic platform Lego® Mindstorms, whose main application is that of the balls that are located in the periphery of the court during games between two people (singles), or more competitors (doubles). The goal of the system is to prevent players from becoming a place to pick up the balls that are on the court, and where the robot is from a distance. For the development of the project, a coding of the system was included in the programming language provided by the LabVIEW@ software of the North American company National Instruments.

\footnotetext{
1 Ingeniero Mecánico, Universidad INCCA, Colombia. MSc. En Ingeniería - Automatización Industrial, Universidad Nacional, Colombia. Docente Universidad Distrital Francisco José de Caldas, Colombia. Correo electrónico: jaforeroc@udistrital.edu.co. ORCID: https://orcid.org/0000-0002-5884-1350.

2Ingeniero Mecánico, Universidad Distrital Francisco José de Caldas, Colombia. Lugar de trabajo: Ingeniero en Bussines and Quality Services S.A.S., Colombia. Correo electrónico: ingdavidgt1@outlook.com. orCID: https://orcid.org/0000-0003-3480-1917.

3 Ingeniero Mecánico, Universidad Distrital Francisco José de Caldas, Colombia. Lugar de trabajo: Ingeniero en Titan Decko, Ecuador. Correo electrónico: hectormartinez 22@hotmail.com. ORCID: https://orcid.org/0000-0002-6788-9479.

Citar este artículo como: J. A. Forero-Casallas, D. L. Gaitán-Tabares y H. F. Martínez-Arcila, "Recolector autónomo de bolas de tenis mediante visión artificial”, Visión electrónica, algo más que un estado sólido, vol. 1, no. 1, Edición especial, enero-junio 2018. DoI revista: https://doi.org/10.14483/issn.2248-4728.
} 


\section{Introducción}

En las canchas de tenis a menudo se observa que los jugadores deben parar el juego para recoger las pelotas que han quedado fuera de la zona de juego, en otras ocasiones contratan a un caddy para realizar esta labor. Algunas veces los jugadores prefieren horarios nocturnos o lugares de juego que se encuentran en zonas campestres y alejadas de la ciudad, es por ello que resulta difícil conseguir un caddy o los costos para su contratación son elevados.

Se ha visto la necesidad de implementar un robot autónomo cuya función sea recoger las bolas de tenis que se encuentren fuera del campo de juego y llevarlas a una zona destinada para el saque de cada jugador, Chawla [1]. Esto se hizo de manera autónoma, eficiente y sin interrumpir el juego para darle continuidad a los partidos o los entrenamientos y evitar al máximo los tiempos muertos que causa la recolección de bolas de tenis, Tsai [2].

Por lo anterior, se propuso diseñar el prototipo de un dispositivo autónomo para recoger bolas de tenis que se encuentren fuera del área de juego, sin interrumpir las actividades deportivas al interior de ésta, para llevarlas a una zona especificada y de esta forma evitar pérdidas de tiempo de los jugadores y sobrecostos en sus horas de juego

\section{Materiales y Métodos}

El objetivo principal del proyecto es desarrollar un robot autónomo para recolectar bolas de tenis con la ayuda de un sistema de visión artificial. El desarrollo de este proyecto se logró mediante la integración de varios pasos fundamentales para la construcción de un prototipo capaz de recoger pelotas de tenis ubicadas fuera del campo de juego y llevarlas a una zona especificada para dejarlas allí y que los jugadores puedan hacer uso de ellas. El dispositivo debe ser autónomo en el sentido de que la programación del sistema de visión artificial permita la ubicación de las bolas que se encuentran en la cancha, y seleccione las que están fuera del área de juego determinando cuál es la más cercana al robot, seguido a esto el prototipo se accionará por el usuario, realizando el recorrido para la recolección de la bola; cada vez que el operador ejecute el programa tomando una nueva fotografía, se actualizarán las coordenadas de la pelota más cercana al equipo.

Todos los sistemas automatizados se componen de tres partes fundamentales, sensores que envían señales percibidas en el medio, procesadores que realizan la tarea de pensar y tomar decisiones según lo que se requiera, y actuadores que ejecutan la tarea para la que se diseña el sistema, Romero [3].

\section{Prototipo Propuesto}

Fue desarrollado con la ayuda del dispositivo Mindstorms Ev3 que fabrica la compañía danesa

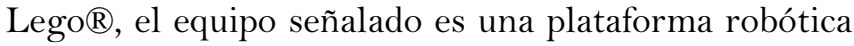
programable que trae un lenguaje propio de programación conocido en sus primeras versiones como Robolab, dicho software es proporcionado a la compañía europea por National Instruments; dado que la compañía que provee el software a Lego es la misma que fabrica LabVIEW, este último posee un complemento que lo hace completamente compatible con el Ev3.

El mecanismo de recolección diseñado con las piezas desmontables del mindstorms se basa en una pinza accionada por un motor mediano, con el que se consigue un movimiento de un grado de libertad (GDL); adicionalmente todo el dispositivo está montado en un sistema de tracción que emplea orugas para un mejor agarre sobre cualquier superficie.

Como el prototipo funciona con visión artificial se requiere de un sensor que capte imágenes continuas (Video) ya que se debe conocer la posición que tiene el robot sobre la cancha en tiempo real, en consecuencia, se seleccionó y empleó una cámara web estándar con sensor CCD Logitech de alta definición.

Finalmente, y una de las cosas más importantes a la hora de diseñar un robot total o parcialmente autónomo, es lograr reproducir la capacidad humana de pensar y tomar decisiones, para este caso la inteligencia artificial y el procesamiento de las señales enviadas desde la cámara se hacen con la ayuda del software LABVIEW® en un equipo portátil Asus K550Dß.

\section{Canchas de prueba}

Dado el tamaño del prototipo y teniendo en cuenta la cantidad de pruebas que se debían realizar para conseguir un dispositivo completamente funcional, se decidió trabajar en una cancha a escala, la relación proporcional entre una de tamaño verdadero y la construida es de 1:11. Las dimensiones de la cancha fueron tomadas del reglamento oficial provisto por la ITF (International Tennis Federation) que indica lo siguiente: La cancha será un rectángulo de 23,77 m de largo por 8,23 $\mathrm{m}$ de ancho. Para los partidos de dobles la pista será de 10,97 m, Bal [4].

La pista estará dividida en su mitad por una red suspendida de una cuerda o un cable metálico cuyos extremos estarán fijados a la parte superior de dos postes o pasarán sobre la parte superior de dos postes a 
una altura de 1,07 m. La red estará totalmente extendida de manera que llene completamente el espacio entre los dos postes de la red y la malla debe ser de un entramado lo suficientemente pequeño para que no pase la pelota de tenis. La altura en el centro de la red será de 0,914 m, en donde estará sostenida mediante una faja. Habrá una banda cubriendo la cuerda o el cable metálico y la parte superior de la red. La faja y la banda serán blancas por todas partes.
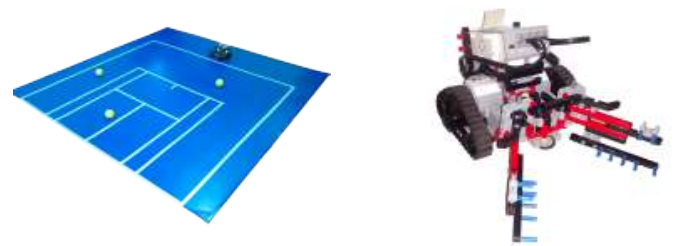

Figura 1. Área de trabajo y Robot recolector. Fuente: elaboración propia

\subsection{Sistema de visión}

En este caso la cámara que es el sensor principal y componente fundamental del sistema de visión artificial, se situó a una altura de 2,5 m paralela al suelo. La conexión del dispositivo de video a la unidad de procesamiento se hizo de forma alámbrica mediante un cable USB, y la vinculación del dispositivo recolector al computador se efectuó de forma inalámbrica utilizando conectividad bluetooth.

\subsection{Programación}

La programación del dispositivo fue realizada en el lenguaje ofrecido por el software LabVIEW® de National Instruments aprovechando la compatibilidad que tiene con el dispositivo de hardware utilizado (Lego Mindstorms Ev3), y se estructuro de la siguiente manera:

-Adquisición de la Imagen.

- Captura de la imagen

- Procesamiento de la Imagen y definición de patrones.

-Determinación de sistema de coordenadas bidimensionales.

- Programación de Inteligencia Artificial.

- Programación de movimiento en los servomotores

-Diseño de Interfaz de Usuario.

\subsection{Interfaz de usuario}

La interfaz gráfica de usuario fue desarrollada pensando en la simplicidad máxima que podría tener el software, para ello se utilizaron solamente indicadores que den a conocer la información necesaria, y los botones de ejecución del programa.

Como se evidencia en la Figura 2, el panel de usuario presenta en la zona izquierda de la pantalla dos botones: uno que captura una imagen con el fin de ubicar las pelotas en el campo de juego, procesar la información y tomar la decisión, y otro botón que ordena al robot ejecutar la tarea de recoger la pelota seleccionada.

En la parte derecha se encuentra un conjunto de indicadores que muestran las distancias (en centímetros), que hay desde la posición del robot hasta la pelota que debe recoger; y la distancia desde dicha bola a la zona de partida del dispositivo, de tal manera que el sistema siempre llegara a la región de inicio definida sin importar donde se encuentre.

Finalmente, en el centro se encuentra una pantalla virtual que muestra el movimiento en tiempo real del robot, y debajo de la misma un indicador de nivel de carga de las baterías del equipo, para conocer el estado de las mismas. La interfaz gráfica fue diseñada con un tema alusivo al tenis en tonos de contraste verde que le da un aspecto visualmente atractivo.

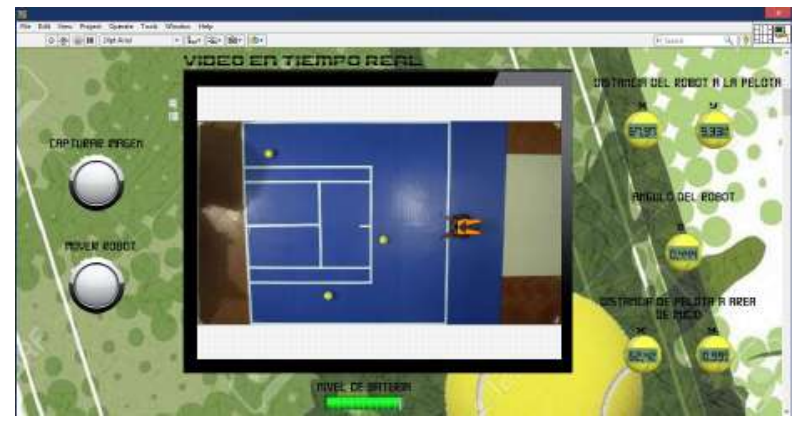

Figura 2. Software de control para el sistema de recolección. Fuente: elaboración propia

El diseño es un sistema autónomo cuya programación permite la ubicación de las bolas fuera del campo de juego y posteriormente realiza el recorrido más óptimo para su recolección. Esto se logró con la ayuda de un sistema de visión artificial que está envía información actualizada al dispositivo de control del robot para asignar una rutina, de tal forma que ésta se haga de la forma más eficiente posible.

El robot está en la capacidad de realizar la labor para la cual fue diseñado, calculando la posición de la pelota mediante el sistema de visión artificial y desplazándose hasta ésta de manera autónoma después de la activación desde la interfaz gráfica del usuario. 
El sistema de inteligencia artificial es capaz de seleccionar la pelota más cercana al robot para que ésta sea su primer objetivo. Además, identifica si la bola se encuentra en la zona de restricción donde el dispositivo no puede entrar, dada dicha situación el robot no realiza ninguna acción hasta que haya una pelota en la zona hábil de recolección.

Se hizo un prototipo en plataforma LEGO®, útil para realizar su labor de recolección de pelotas de tenis en la cancha de pruebas, en el cual se diseñaron los siguientes sistemas: un tren de rodaje tipo oruga, una pinza con 1 grado de libertad para sujetar la pelota, un patrón geométrico de reconocimiento y un bastidor sobre el cual se montaron los anteriores elementos.

\section{Validación}

Éstas, son pruebas a las cuales se sometió el prototipo para tomar muestras de datos, sacando a partir de ellos resultados estadísticos que permitieron concluir si el robot estaba en la capacidad de cumplir la función para la cual fue diseñado en la cancha de pruebas. Dichos ensayos fueron útiles para hacer mediciones reales y proyectar el prototipo a posibles cambios futuros que contribuyan a mejorar las características técnicas del dispositivo, su funcionalidad y eficiencia.

Algunos proyectos basados en visión artificial y robótica han sido sometidos a pruebas de validación y experimentos en tiempo real, con el fin de hacer mediciones y validar los equipos determinando qué tan funcionales son y cómo se pueden mejorar. Uno de los proyectos en los cuales se recurrió a este tipo de pruebas fue en titulado: "A visión based control platform for Industrial Robot Rehabilitation" desarrollado en la Universidad Autónoma del Estado de Morelos en la ciudad de México, donde se hicieron pruebas de validación del controlador implementado y se llevaron a cabo experimentos en tiempo real con el control basado en visión. Las tres partes fundamentales de cada prueba fueron: selección del punto objetivo, posicionamiento del manipulador y medición del error.

Otro trabajo en el cual se involucraron pruebas para validar robots fue el titulado: "ROSPHERE, Diseño, Construcción y Aplicación de una Esfera Robótica”; el autor del proyecto, Juan David Hernández Vega, desarrolló un prototipo con un sistema de tracción no convencional y lo sometió a un conjunto de 4 pruebas que permitieron validar las características del robot, éstas fueron: validación de movimientos y adquisición sensorial, interacción segura con personas, versatilidad y adaptabilidad a diferentes terrenos y desempeño global en tareas de monitorización de variables ambientales.

En el presente trabajo, se diseñaron cuatro pruebas para hacer la validación del prototipo. La cancha se dividió en tres zonas, tomando datos y obteniendo resultados para cada una. El motivo por el cual se hizo este seccionamiento, fue por la necesidad de saber cuáles eran las regiones críticas, en función de la posición de la cámara, su resolución y la distancia hasta el área de salida del robot; de esta forma se obtuvo información de las zonas a las cuales se debía prestar especial atención para la programación del equipo y si el prototipo era funcional para operar en la cancha de pruebas.

\section{Resultados esperados}

El resultado principal de este trabajo consiste en diseñar e implementar un sistema autónomo para recolección de bolas de tenis mediante visión artificial.

\section{Discusión}

Hoy en día los avances tecnológicos son uno de los fenómenos que mueven el mundo y motivan la creatividad y el talento de la humanidad. Estar a la vanguardia de la tecnología se ha convertido en una de las herramientas más importantes del ingeniero.

El tenis es un deporte de gran acogida a nivel mundial, el cual llama la atención de muchas personas y las motiva a practicarlo, bien sea a nivel profesional o como hobby. En el campo de juego los tenistas a menudo interrumpen el juego para recoger las bolas de tenis o designan a una persona que esté realizando esta labor de recogerlas y llevarlas a la zona de servicio. En algunas ocasiones los deportistas aficionados que practican este deporte no consiguen un recogebolas bien sea por el horario de juego, el lugar donde se encuentra la cancha o simplemente por los elevados costos que implica la contratación de una persona que realice esta labor. Este proyecto soluciona y facilita todos estos inconvenientes. El robot está en la capacidad de realizar la labor para la cual fue diseñado, calculando la posición de la pelota mediante el sistema de visión artificial y desplazándose hasta ésta de manera autónoma después de la activación desde la interfaz gráfica del usuario.

El sistema de inteligencia artificial es capaz de seleccionar la pelota más cercana al robot para que ésta sea su primer objetivo. Además, identifica si la bola se encuentra en la zona de restricción donde el dispositivo no puede entrar, dada dicha situación el robot no realiza ninguna acción hasta que haya una pelota en la zona hábil de recolección.

Se implementó un algoritmo de calibración del equipo en algunos movimientos, de tal forma que autónomamente el dispositivo se corrija cuando el 
sistema de visión artificial detecte que no seguirá la trayectoria adecuada.

Se hizo un prototipo en plataforma LEGO $®$, útil para realizar su labor de recolección de pelotas de tenis en la cancha de pruebas, en el cual se diseñaron los siguientes sistemas: un tren de rodaje tipo oruga, una pinza con 1 grado de libertad para sujetar la pelota, un patrón geométrico de reconocimiento y un bastidor sobre el cual se montaron los anteriores elementos. El robot es capaz de desplazarse a una velocidad promedio de 0,15 $\mathrm{m} / \mathrm{s}$.

De acuerdo a los resultados obtenidos en las pruebas de validación el robot tiene una eficacia del $90 \%$.

Sin importar que el equipo se encuentre desviado del área de salida, el sistema de visión artificial puede detectar la posición relativa entre el robot y la pelota para su posterior recolección.

Según los resultados de las pruebas de validación el sistema de visión y el prototipo operan con un error permisible de las coordenadas calculadas y la llegada a la pelota respectivamente, sin embargo, el sistema realiza su labor adecuadamente.

\section{Referencias}

[1] N. Chawla, W. Alwuqayan, A. Faizan y S. Tosunoglu, "Robotic Tennis Ball Collector", Conference on Recent Advances in Robotics, Florida International University, 2012. pp. 1-6.

[2] J. Tsai, "Robot for collecting table tennis balls". United States Patent. Taipei (TW).2010.

[3] F. Romero, M. Vargas, L. Castaño. "Integración De Sistemas De Visión Artificial”. Escuela Superior de Ingenieros de Sevilla. España. 2006.

[4] B. Bal, G. Dureja. "Hawk Eye: A Logical Innovative Technology Use In Sports for Effective Decision Making". Sport Science Review, Varsovia, 2012. 